\title{
An Approach for Measuring the Dielectric Strength of OLED Materials
}

\author{
Sujith Sudheendran Swayamprabha, Deepak Kumar Dubey, Wei-Chi Song, You-Ting Lin, \\ Rohit Ashok Kumar Yadav, Meenu Singh and Jwo-Huei Jou*
}

Department of Materials Science and Engineering, National Tsing Hua University, Hsinchu 30013, Taiwan; sujithsudheendran.s.s@gmail.com (S.S.S.); deepak08gkp@gmail.com (D.K.D.);

ahenry94125@gmail.com (W.-C.S.); zaa789p2002@hotmail.com (Y.-T.L.); rohitakyadav@gmail.com (R.A.K.Y.); meenu9696@gmail.com (M.S.)

* Correspondence: jjou@mx.nthu.edu.tw

Received: 7 May 2018; Accepted: 6 June 2018; Published: 9 June 2018

\begin{abstract}
Surface roughness of electrodes plays a key role in the dielectric breakdown of thin-film organic devices. The rate of breakdown will increase when there are stochastic sharp spikes on the surface of electrodes. Additionally, surface having spiking morphology makes the determination of dielectric strength very challenging, specifically when the layer is relatively thin. We demonstrate here a new approach to investigate the dielectric strength of organic thin films for organic light-emitting diodes (OLEDs). The thin films were deposited on a substrate using physical vapor deposition (PVD) under high vacuum. The device architectures used were glass substrate/indium tin oxide (ITO)/organic material/aluminum (Al) and glass substrate/Al/organic material/Al. The dielectric strength of the OLED materials was evaluated from the measured breakdown voltage and layer thickness.
\end{abstract}

Keywords: Breakdown voltage; Dielectric strength; OLED

\section{Introduction}

Organic light-emitting diodes (OLEDs) have drawn enormous attention in both academia and industry owing to their cumulative applications in high-quality flat-panel displays and solid-state lighting [1-6]. Recently, OLEDs have been commercialized as information displays for car audio systems, sub-displays of cellular phones, and large-screen TVs, promising large market opportunities [7-10]. The superior properties of OLEDs start to dominate existing flat-panel display technology, liquid-crystal display (LCD). OLEDs have striking topographies, such as high color purity, high luminance, a wide viewing angle, high contrast and response speed, low power consumption, a simple fabrication process, ultra-thin structure, light weight, flexibility, and low cost [11]. To make OLED displays and lighting more competitive and customer affordable, and the resultant products more energy saving and longer lasting, OLEDs with higher power efficiency are demanded [3].

In the last two decades, state-of-the-art OLEDs have achieved prodigious progress in efficiency, making their potential applications exceedingly promising [3]. However, lifespan is still a crucial reliability issue to be addressed before they can be extensively adopted. Light-emitting diodes based on both inorganic and organic semiconducting materials suffer from internal energy losses, and these losses convert into heat [12]. The produced heat can then itself act as a source of degradation and decrease the lifetime of the device. Therefore, reduction of heat and dissipation is required in order to increase the lifetime of OLEDs [13]. In addition, device failures and lifetime are affected by several other factors, such as electrochemical degradation, oxidation, moisture, molecular migrations, 
and dielectric breakdown [12]. Surface roughness of the electrodes plays a major role in dielectric breakdown [14].

Only a few reports are available regarding the dielectric properties of OLED materials. In 1993, Nguyen et al. analyzed frequency-dependent capacitive response in indium tin oxide (ITO)/poly(p-phenylene vinylene) (PPV)/aluminum (Al) devices, such as conductance and tan $\delta$ [15]. Li et al. studied the alternating current impedance of light-emitting diodes (LED) and light-emitting electrochemical cells (LEC), and propsed an equivalent circuit with a series combination of series resistance $\left(\mathrm{R}_{0}\right)$ and parallel resistor-capacitor $(\mathrm{RC})$ [16]. Even though the structure of OLEDs has a single layer, it shows two semicircles in Cole-Cole plot. Wang et al. reported that materials with high dielectric constant can perform as a good electron injection layer (EIL), which enhanced device efficiency and brightness [17]. Ohta et al. studied the influence of high dielectric strength materials in an active matrix driving OLEDs [18]. Ahn et al. examined the magnitude and phase of impedance, electrical conductivity, and dielectric loss under different biasing voltage in OLEDs [19].

Generally, OLED devices have a driving voltage of 3 to $5 \mathrm{~V}$. Depending on the color of the light and the thickness of organic layers, OLED devices need higher voltage in order to achieve more brightness. However, device efficiency and lifetime start to decline at a higher driving voltage. This failure of OLED devices is mainly due to dielectric breakdown of the organic material. For a thin film of $100 \mathrm{~nm}$, a voltage of $10 \mathrm{~V}$ can produce an electric field of 1 million volts $/ \mathrm{cm}(\mathrm{MV} / \mathrm{cm})$. The produced electric field is sufficient to cause dielectric breakdown of most of the OLED materials [20]. In this study, we attempted to discover the correlation between surface roughness of the electrode and dielectric breakdown of OLED materials. We measured the dielectric strength of the OLED materials by evaluating the breakdown voltage and layer thickness [21].

\section{Experimental Section}

\subsection{Materials}

Indium tin oxide (ITO) coated glass substrates with a sheet resistance of $15 \Omega / \mathrm{sq}$, surface roughness of $1.5 \mathrm{~nm}$, and light transmittance greater than $84 \%$ were purchased from Luminescence Technology Corporation, Hsinchu, Taiwan. The molecular structures of the organic materials used in this work are shown in Figure 1. The sublimated grade organic materials 4,7-Diphenyl-1,10-phenanthroline (BPhen) and 4,4'-Bis(9-carbazolyl)-1,1'-biphenyl,4,4-N,N $N^{\prime}$-Dicarba zole-1,1'biphenyl (CBP) were purchased from the Luminescence Technology Corporation and Wang Shine Co., Taichung, Taiwan, respectively. Aluminum (Al) ingots $(99.999 \%)$ were purchased from Showa Chemical Co. Ltd., Tokyo, Japan. All materials were used without any further purification.

(a)

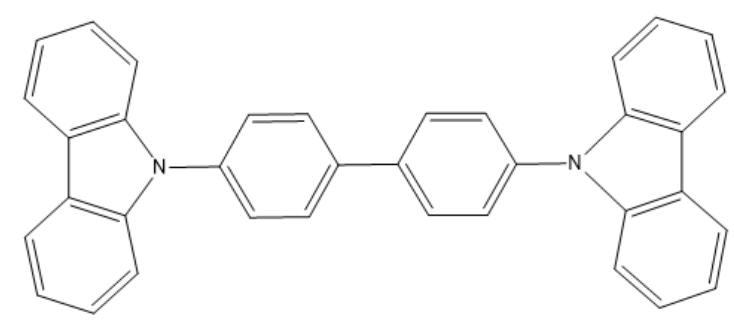

(b)

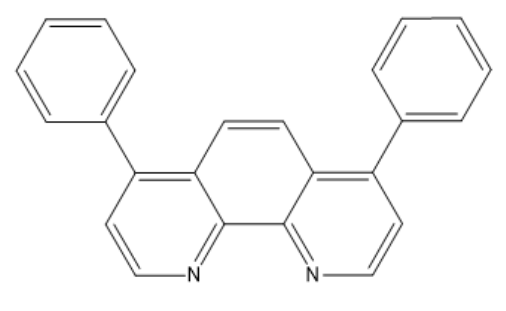

Figure 1. Molecular structures of (a) 4,4'-Bis(9-carbazolyl)-1, $1^{\prime}$-biphenyl,4,4- $N, N^{\prime}$-Dicarba zole-1,1'biphenyl (CBP) and (b) 4,7-Diphenyl-1,10-phenanthroline (BPhen).

\subsection{Device Fabrication}

The study of the dielectric properties of OLED devices was performed on the organic layer sandwiched between two electrodes, with the device structure metal/organic layer/metal (MOM). The devices were grown on glass slides pre-coated with ITO. The ITO substrates were ultra-sonicatied 
sequencially in acetone and isopropanol for $30 \mathrm{~min}$, followed by $20 \mathrm{~min}$ of ultraviolet ozone treatment to eliminate all organic impurities. Device I was fabricated with an architecture of Glass/ITO/CBP/Al; Device II: Glass/Al/CBP/Al; Device III: Glass/ITO/BPhen/Al; and Device IV: Glass/Al/BPhen/Al. All materials were subsequently deposited on the substrates by thermal evaporation under high vacuum $\left(<10^{-6}\right.$ torr). Device I comprised of a $1250 \AA$ ITO anode, $1200 \AA$ CBP, and a $1000 \AA$ aluminum cathode. Device II comprised of a $1250 \AA$ aluminum anode, $1200 \AA \mathrm{CBP}$, and a $1000 \AA$ aluminum cathode. Device III and Device IV followed the same thickness of the materials of Device I and Device II, respectively, but replacing CBP with BPhen. All thickness measurements were done by alpha step (Dektak 150, Veeco Instruments, Inc., Plainview, NY, USA) where possible instrumental error may be $\pm 5 \mathrm{~nm}$. Purity of the deposited organic materials CBP and BPhen were confirmed by proton nuclear magnetic resonance $\left({ }^{1} \mathrm{HNMR}\right.$, Bruker Avance $400 \mathrm{NMR}$ spectrometer, Bruker Corp., Billerica, MA, USA), as shown in Figures S1 and S2, respectively.

\section{Measurements}

Surface morphology was studied using an atomic force microscope (Digital Instruments Nanoscope IIIa, Bruker Corp., Billerica, MA, USA) in the tapping mode. A Keithley 2400 electrometer (Keithley Instruments, Inc., Cleveland, OH, USA) was used to measure the current-voltage (I-V) characteristics. All electrical characterizations have been done under open environment at room temperature.

From the breakdown voltage, we calculated the dielectric strength of the material using the equation:

$$
E=V / t
$$

where, $E, V$, and $t$ are the dielectric strength, breakdown voltage in $\mathrm{MV}$, and deposited layer thickness in $\mathrm{cm}$, respectively.

\section{Result and Discussion}

Figure 2a,b shows the studied devices composed with glass/ITO/organic material/Al and glass/ $\mathrm{Al} /$ organic material/ $\mathrm{Al}$ for dielectric strength measurement, respectively. Figure $2 \mathrm{c}, \mathrm{d}$ illustrates the respective electric circuits of studied devices. Figure $3 a, b$ shows the breakdown voltage of Device I and Device II. Device I showed a breakdown voltage of $3.90 \mathrm{~V}$, whereas Device II showed 6.20 V. The calculated dielectric strengths were 0.32 and $0.52 \mathrm{MV} / \mathrm{cm}$ for Device I and Device II, respectively.

Similarly, Figure 4a,b shows the breakdown voltages of Device III and Device IV. Device III showed a lower breakdown voltage when compared with Device IV. The breakdown voltages were 7.52 and $13.80 \mathrm{~V}$ for Device III and Device IV, respectively. The calculated dielectric strengths were 0.63 and $1.15 \mathrm{MV} / \mathrm{cm}$ for Device III and Device IV, respectively. The dielectric strength results are summarized in Table 1.

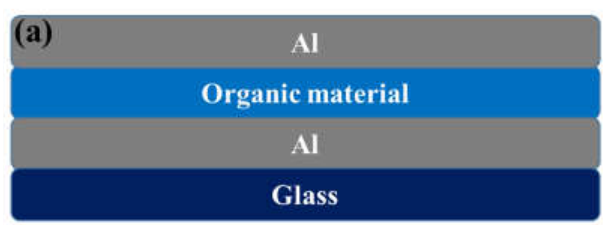

(c)

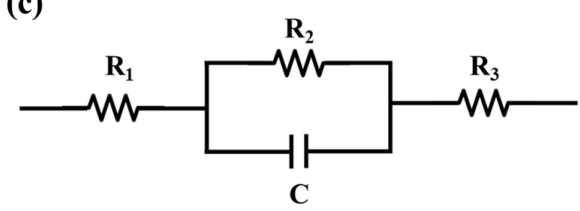

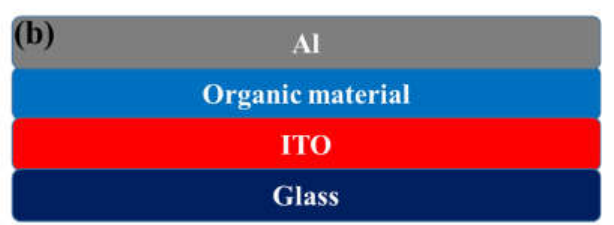

(d)

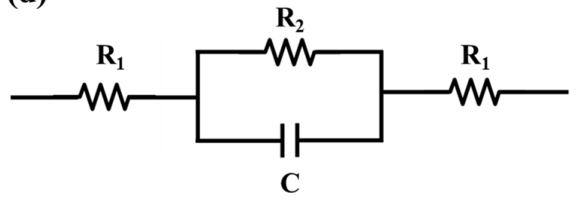

Figure 2. $(\mathbf{a}, \mathbf{b})$ show the studied device architectures for the dielectric strength measurements and (c,d) show their respective electrical circuit diagrams. 

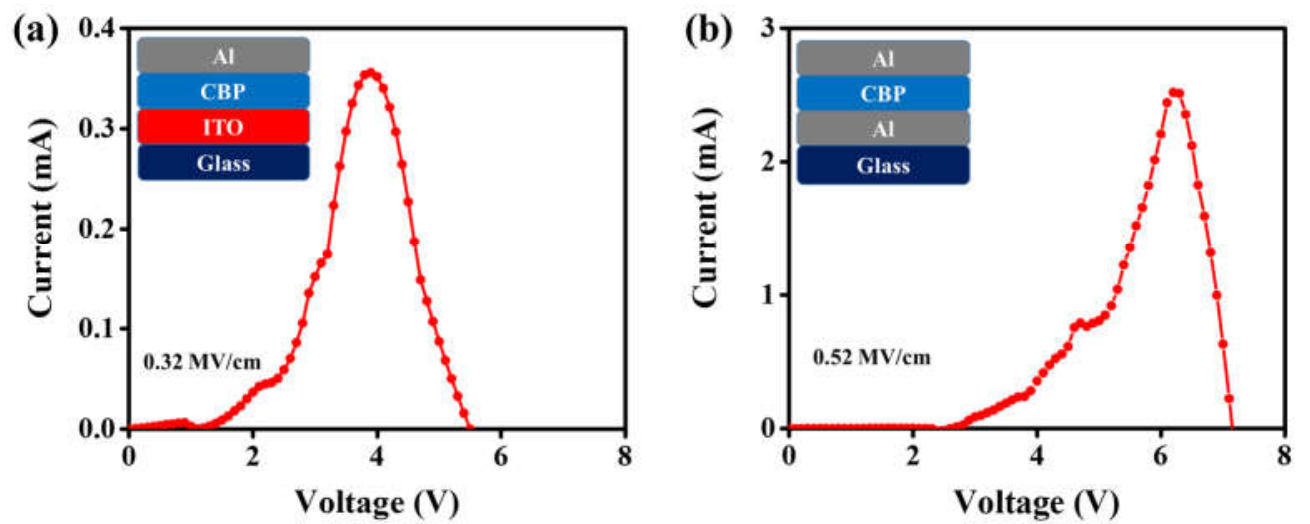

Figure 3. Current-Voltage (I-V) characteristics of the devices having $120 \mathrm{~nm}$ CBP layer with (a) ITO/CBP/aluminum ( $\mathrm{Al})$ and (b) $\mathrm{Al} / \mathrm{CBP} / \mathrm{Al}$ device structures.
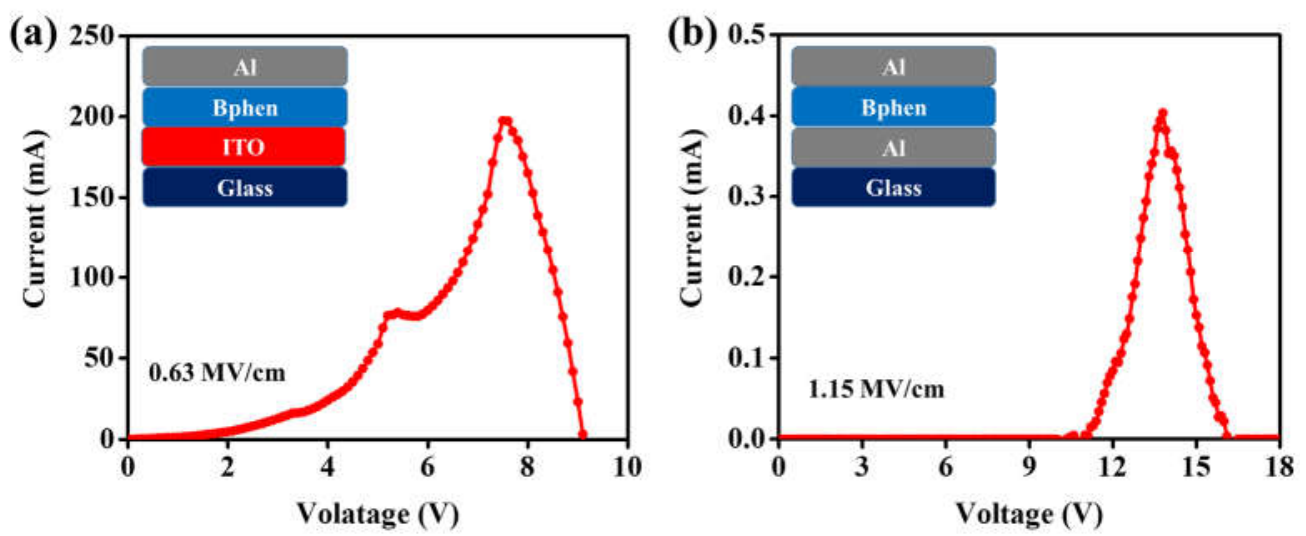

Figure 4. Current-voltage (I-V) characteristics of the devices having a $120 \mathrm{~nm}$ BPhen layer with (a) $\mathrm{Al} / \mathrm{BPhen} / \mathrm{Al}$ and (b) ITO/BPhen/ $\mathrm{Al}$ device structures.

The reason why devices fabricated with an ITO anode show low dielectric strength when compared with devices with an $\mathrm{Al}$ anode may be attributed to two important factors in the designed device architecture. First, the $\mathrm{Al}$ anode has a low work function $(4.3 \mathrm{eV})$ as compared to the ITO anode $(5.2 \mathrm{eV})$. Second, the devices fabricated with an ITO anode more effectively transfer holes into the organic layer because of 0.8 and $1.1 \mathrm{eV}$ hole-injection barriers at the interface of ITO/CBP and ITO/BPhen, respectively, i.e., 1.7 and $2.0 \mathrm{eV}$ for the devices with $\mathrm{Al}$ as an anode, as shown in Figure $5 \mathrm{a}, \mathrm{b}$ and Figure $6 \mathrm{a}, \mathrm{b}$. Furthermore, we observed the low charge injection region in Figures $3 \mathrm{~b}$ and $4 \mathrm{~b}$, below the voltage $2 \mathrm{~V}$ and $10 \mathrm{~V}$, for Devices II and IV, respectively. That current density (J) follows the externally applied voltage (V) linearly, as shown in Equation (2), may be the reason behind this [22].

Table 1. Tabulation of the used anode, observed breakdown voltage, and calculated dielectric strength for the deposited $120 \mathrm{~nm}$ thickness of the organic layer.

\begin{tabular}{cccc}
\hline Organic Material & Anode & Breakdown Voltage (V) & Calculated Dielectric Strength (MV/cm) \\
\hline CBP & ITO & 3.90 & 0.32 \\
CBP & Al & 6.20 & 0.52 \\
Bphen & ITO & 7.52 & 0.63 \\
Bphen & Al & 13.80 & 1.15 \\
\hline
\end{tabular}



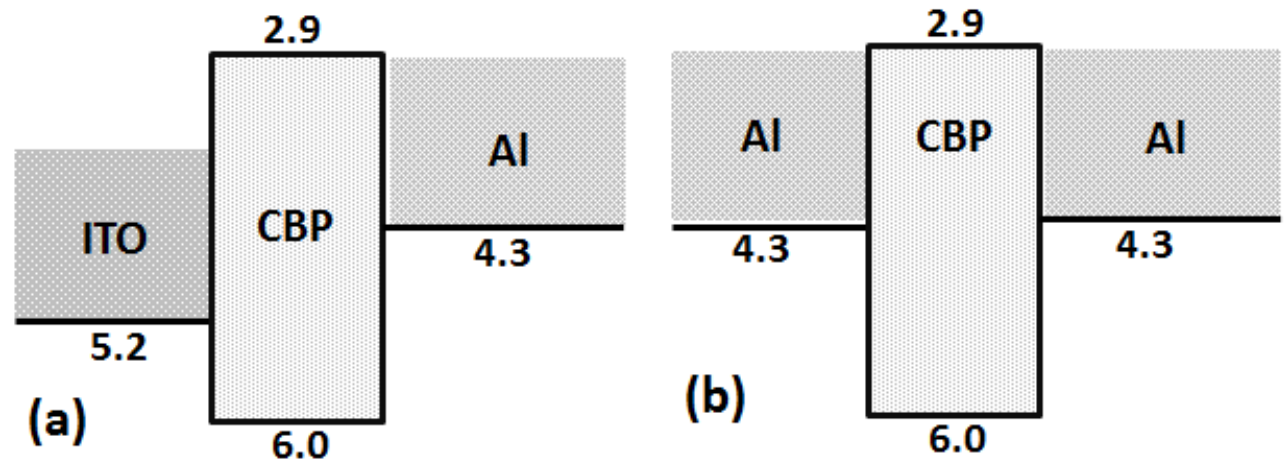

Figure 5. Schematic energy diagram of devices with the following device structure: (a) ITO/CBP/Al; and (b) $\mathrm{Al} / \mathrm{CBP} / \mathrm{Al}$.
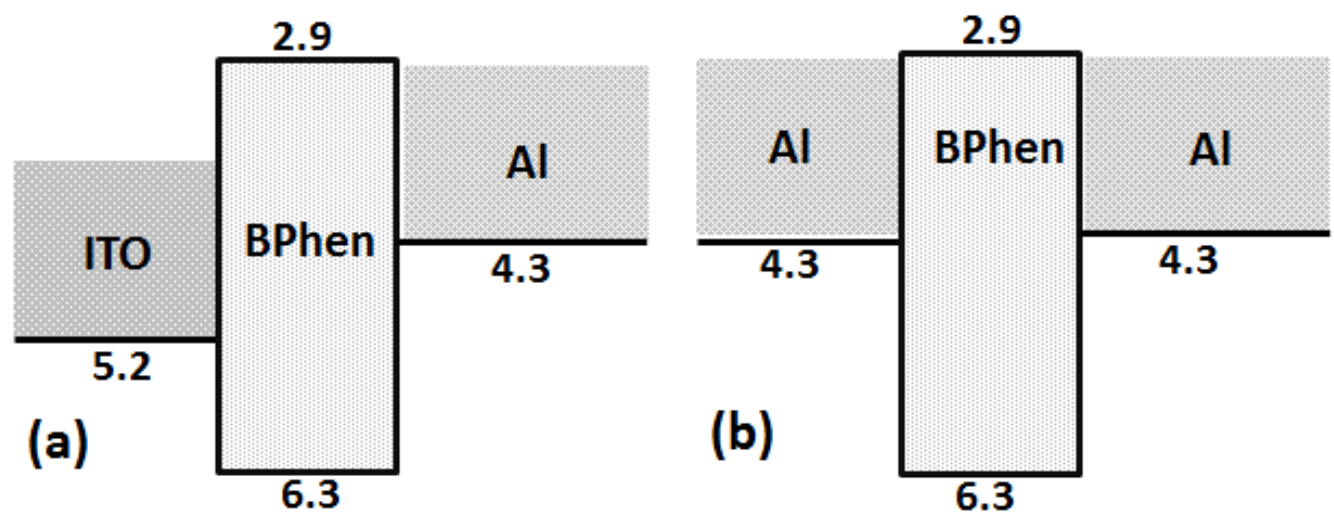

Figure 6. Schematic energy diagram of devices with the following device structure: (a) ITO/BPhen/ $\mathrm{Al}$; and (b) $\mathrm{Al} / \mathrm{BPhen} / \mathrm{Al}$.

$$
J=e n u_{e f f} \frac{V}{L}
$$

where $e n$ is the charge density (e: elementary charge, $n$ : charge carrier density), $\mu_{\text {eff }}$ is the effective charge mobility, and $L$ the organic film thickness. It is notable that the effective charge mobility $\mu_{\text {eff }}$ includes charge trapping phenomenon as follows [22]:

$$
u_{e f f}=u_{0}\left(\frac{n_{m o b}}{n_{m o b}+n_{\text {trap }}}\right)
$$

where $\mu_{0}$ is trap-free charge mobility, and $n_{\text {mob }}$ and $n_{\text {trap }}$ are mobile and trapped charge carrier densities, respectively. As shown in Figure 3b, in the voltage region from 2 to $3 \mathrm{~V}$ an abrupt increase of the current is observed. Note that the significant rise of the current in certain voltage regions only is usually assigned to the trap-filled-limit (TFL) voltage. In other words, the charge transport properties of organic films are changed due to filling of all localized states, and charge carriers are no more influenced by the trapping mechanism. Similar behavior is also observed in Device IV in the voltage region from 10 to $12 \mathrm{~V}$, as shown in Figure $4 \mathrm{~b}$.

In addition, the devices with BPhen as an organic layer show higher dielectric strength as compared to devices with CBP organic layers. The reasons behind this may be: (i) highest occupied molecular orbital (HOMO) level of BPhen is deeper (6.3 eV); (ii) high electron mobility of BPhen; and (iii) the bipolar nature of CBP [23-26].

The surface topography of the pristine ITO and thermally deposited Al were studied using atomic force microscopy (AFM) in the tapping mode. AFM data analysis provides quantitative information 
about surface morphology. Two-dimensional (2D) and three-dimensional (3D) AFM micrographs of the pristine ITO and thermally deposited Al (Figure 7) samples clearly show that the obtained films indeed possess a uniform surface, very developed grain boundaries, and free surfaces. Roughness of the films was estimated with Nanoscope analyser. Scans over $4 \times 4 \mu \mathrm{m}^{2}$ were taken in order to measure the surface roughness of the films. The root-mean square roughness $\left(R_{R M S}\right)$ was estimated by using the formula [27]:

$$
R_{R M S}=\sum_{i=1}^{N}\left(\frac{\left(h_{i}-\hbar\right)^{2}}{N}\right)^{1 / 2}
$$

where $h_{i}$ and $\hbar$ represents the height value at each data point and the profile mean value of the surface, respectively, and $N$ is the number of data points in the analyzed profile. The standard roughness, or the arithmetic average roughness -height, represents the arithmetic mean of the deviations in height from the profile mean value, where the profile mean value is defined as [27]:

$$
\hbar=\frac{1}{N} \sum_{i=1}^{N} h_{i}
$$

The $R_{R M S}$ estimated for the films of thermally deposited $\mathrm{Al}$ and pristine ITO were 2.5 and $3.6 \mathrm{~nm}$, respectively. We could correlate the variation in dielectric strength with the surface roughness of both ITO and Al. The dielectric strength of materials was inversely proportional to the surface roughness of the anode. The reason why devices with an ITO anode show lower dielectric strength as compared to devices with an $\mathrm{Al}$ anode may also be attributed to the low surface roughness of $\mathrm{Al}$ as compared to ITO.
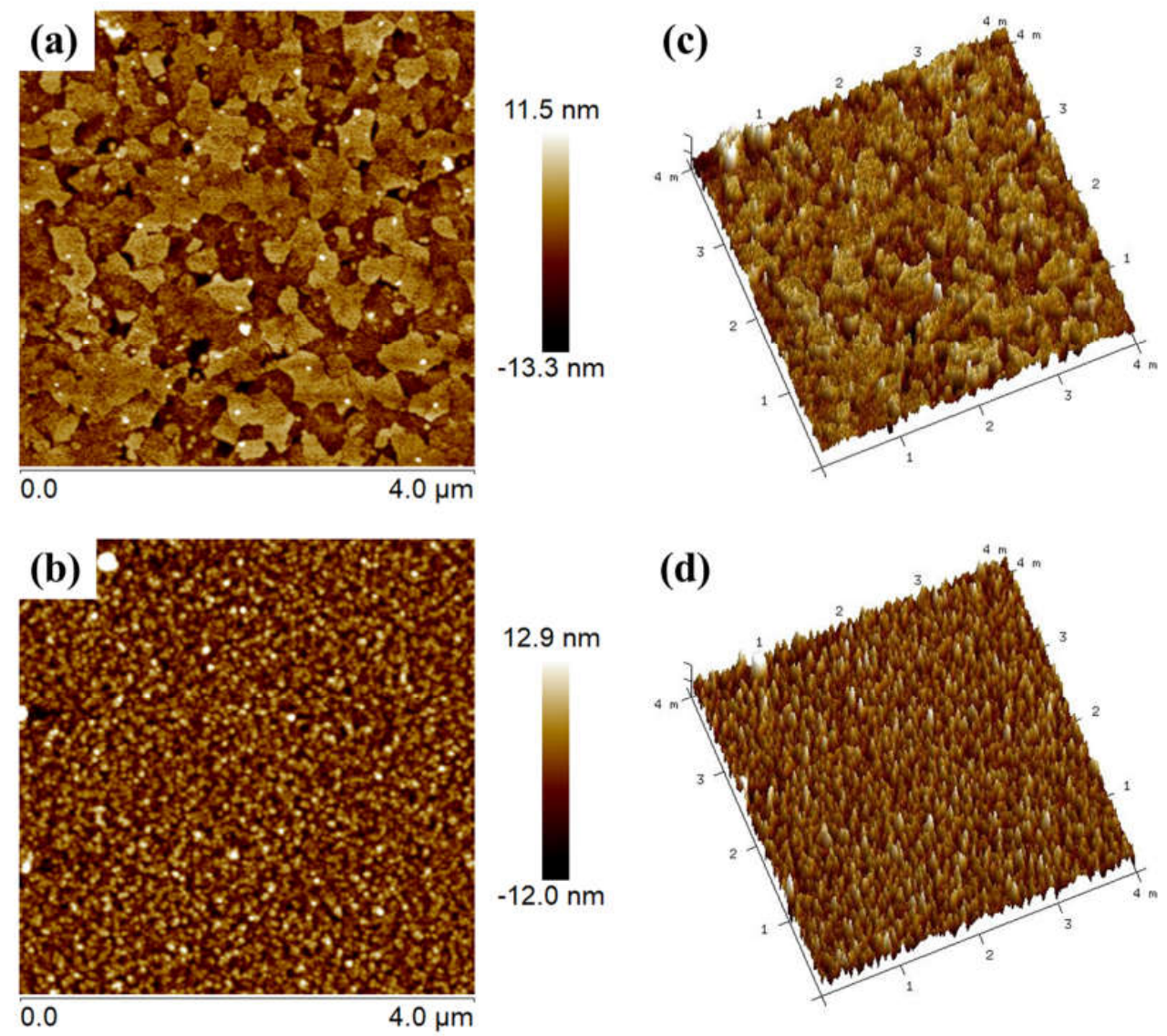

Figure 7. (a,b) show the 2D atomic force micrographs of the ITO and thermally deposited Al anode surface, respectively; (c,d) show their respective 3D micrograps. 


\section{Conclusions}

In this study, the dielectric properties of OLED materials were investigated using metal/organic layer/metal device structures. The surface roughness of electrodes and dielectric strength of organic materials play a crucial role in dielectric breakdown, which is directly related to the performance of OLED devices. Surface roughness of electrodes will accelerate the dielectric breakdown. By optimizing the surface roughness of electrodes and the dielectric strength of organic materials, we can design and fabricate OLEDs with long lifetime and high-efficiency. Despite various advantages in OLEDs, a fundamental research of their physical properties is not yet been fully explored. Our findings might help domain experts carry out extensive studies to design high-efficiency and long lifespan devices.

Supplementary Materials: The following are available online at http:/ /www.mdpi.com/1996-1944/11/6/979/s1, Figure S1: ${ }^{1} \mathrm{H}$ NMR analysis of 4,4'-Bis(9-carbazolyl)-1,1'-biphenyl,4,4- $N, N^{\prime}$-Dicarbazole-1,1'biphenyl (CBP). Figure S2: ${ }^{1} \mathrm{H}$ NMR analysis of 4,7-Diphenyl-1,10-phenanthroline (BPhen).

Author Contributions: J.-H.J. and S.S.S. conceived and designed the experiments; S.S.S. performed the experiments and analyzed data; W.-C.S., Y.-T.L., R.A.K.Y., and M.S. provided some significant technical inputs; S.S.S. and D.K.D. wrote the paper.

Acknowledgments: This work was financially supported in part by Grants MEA104-EC-17-A-07-S3-012, MOST104-2119-M-007-012, and MOST103-2923-E-007-003-MY3.

Conflicts of Interest: The authors declare that they have no conflict of interests regarding the publication of this paper.

\section{References}

1. Tang, C.W.; VanSlyke, S.A. Organic electroluminescent diodes. Appl. Phys. Lett. 1987, 51, 913-915. [CrossRef]

2. D'Andrade, B.W.; Forrest, S.R. White Organic Light-Emitting Devices for Solid-State Lighting. Adv. Mater. 2004, 16, 1585-1595. [CrossRef]

3. Jou, J.H.; Kumar, S.; Agrawal, A.; Li, T.H.; Sahoo, S. Approaches for fabricating high efficiency organic light emitting diodes. J. Mater. Chem. C 2015, 3, 2974-3002. [CrossRef]

4. Dubey, D.K.; Tung, F.C.; Liu, S.H.; Song, W.C.; Singh, M.; Sahoo, S.; Lai, S.H.; Jou, J.H. P-214: Flexible white organic light emitting diode via solution process. SID Symp. Dig. Tech. Pap. 2017, 48, 2025-2027. [CrossRef]

5. Dubey, D.K.; Singh, M.; Sahoo, S.; Jou, J.H. Simple-structured efficient white organic light emitting diode via solution process. Microelectron. Reliab. 2018, 83, 293-296. [CrossRef]

6. Dubey, D.K.; Yadav, R.A.K.; Tavgeniene, D.; Grigalevicius, S.; Jou, J.H. Crosslinkable hole-transporting small molecule as a mixed host for efficient solution-processed red organic light emitting diodes. Thin Solid Films 2018. [CrossRef]

7. Wu, Z.; Ma, D. Recent advances in white organic light-emitting diodes. Mater. Sci. Eng. R Rep. 2016, 107, 1-42. [CrossRef]

8. Zhao, F.; Ma, D. Approaches to high performance white organic light-emitting diodes for general lighting. Mater. Chem. Front. 2017, 1, 1933-1950. [CrossRef]

9. Jou, J.H.; Swayamprabha, S.S.; Yadav, R.A.K.; Dubey, D.K. Nano-Structures Enabling Sunlight and Candlelight-Style OLEDs. J. Nanomater. Mol. Nanotechnol. 2018, 7. [CrossRef]

10. Borchardt, J.K. Developments in organic displays. Mater. Today 2004, 7, 42-46. [CrossRef]

11. Thejokalyani, N.; Dhoble, S.J. Importance of Eco-friendly OLED Lighting. Defect Diffus. Forum 2014, 357, 1-27. [CrossRef]

12. Scholz, S.; Kondakov, D.; Lüssem, B.; Leo, K. Degradation Mechanisms and Reactions in Organic Light-Emitting Devices. Chem. Rev. 2015, 115, 8449-8503. [CrossRef] [PubMed]

13. Park, J.W.; Lee, J.H. Heat dissipation property of cover glasses with heat sink films for transparent organic light-emitting diodes. Semicond. Sci. Technol. 2014, 29. [CrossRef]

14. Liu, G.; Kerr, J.B.; Johnson, S. Dark spot formation relative to ITO surface roughness for polyfluorene devices. Synth. Matter 2004, 144, 1-6. [CrossRef]

15. Nguyen, T.P.; Tran, V.H.; Massardier, V. Electrical conduction in poly (phenyleneviny1ene) thin films. J. Phys. Condens. Matter 1993, 5, 6243-6252. [CrossRef] 
16. Li, Y.; Gao, J.; Yu, G.; Cao, Y.; Heeger, A.J. ac impedance of polymer light-emitting electrochemical cells and light-emitting diodes: A comparative study. Chem. Phys. Lett. 1998, 287, 83-88. [CrossRef]

17. Wang, F.; Liu, S.; Zhang, C. The dielectric constant of materials effect the property of the OLED. Microelectron. J. 2007, 38, 259-261. [CrossRef]

18. Ohta, S.; Chuman, T.; Miyaguchi, S.; Satoh, H.; Tanabe, T.; Okuda, Y.; Tsuchida, M. Active Matrix Driving Organic Light-Emitting Diode Panel Using Organic Thin-Film Transistors. Jpn. J. Appl. Phys. 2005, 44, 3678-3681. [CrossRef]

19. Ahn, J.H.; Lee, H.S.; Seo, D.S.; Jang, K.U.; Lee, W.J.; Kim, T.W. Dielectric properties depending on frequency in organic light-emitting diodes. Thin Solid Films 2008, 516, 2626-2629. [CrossRef]

20. Kim, H.K.; Shi, F.G. Thickness Dependent Dielectric Strength of a Low-permittivity Dielectric Film. IEEE Trans. Dielectr. Electr. Insul. 2001, 8, 248-252. [CrossRef]

21. Calculating the Dielectric Strength of Material. Available online: https://www.buckleysinternational.com/ pages/calculating-dielectric-stength (accessed on 8 June 2018).

22. Juhasz, P.; Nevrela, J.; Micjan, M.; Novota, M.; Uhrik, J.; Stuchlikova, L.; Jakabovic, J.; Harmatha, L.; Weis, M. Charge injection and transport properties of an organic light-emitting diode. Beilstein J. Nanotechnol. 2016, 7, 47-52. [CrossRef] [PubMed]

23. Naka, S.; Okada, H.; Onnagawa, H.; Tsutsui, T. High electron mobility in bathophenanthroline. Appl. Phys. Lett. 2000, 76, 197-199. [CrossRef]

24. Pereira, A.; Conte, G.; Gallardo, H.; Zucco, C.; Quirino, W.G.; Legnani, C.; Cremona, M.; Bechtold, I.H. OLEDs based on an europium(III) complex: \{Tris(thenoyltrifluoroacetonate)-[1,2,5]thiadiazolo[3,4-f][1,10]phenanthroline\} europium(III). J. SID 2011, 793-797. [CrossRef]

25. Jou, J.H.; Kumar, S.; Singh, M.; Chen, Y.H.; Chen, C.C.; Lee, M.T. Carrier Modulation Layer-Enhanced Organic Light-Emitting Diodes. Molecules 2015, 20, 13005-13030. [CrossRef] [PubMed]

26. Sahoo, S.; Dubey, D.K.; Singh, M.; Joseph, V.; Thomas, K.R.J.; Jou, J.H. Highly efficient deep-blue organic light emitting diode with a carbazole based fluorescent emitter. Jpn. J. Appl. Phys. 2018, 57. [CrossRef]

27. Ulmeanu, M.; Serghei, A.; Mihailescu, I.N.; Budau, P.; Enachescu, M. C Ni amorphous multilayers studied by atomic force microscopy. Appl. Surf. Sci. 2000, 165, 109-115. [CrossRef]

(C) 2018 by the authors. Licensee MDPI, Basel, Switzerland. This article is an open access article distributed under the terms and conditions of the Creative Commons Attribution (CC BY) license (http:/ / creativecommons.org/licenses/by/4.0/). 